\title{
Chronic rhinosinuitis with sphenoid sinus mycetoma - Clinical Case and literature review
}

\author{
Stefan S. Konsulov, K.K. Piskova, Taniel M. Minkov, D. Milkov, S. Konsulov \\ University Hospital „Kaspela“, Department of ENT Diseases, Medical University, Plovdiv
}

\section{Introduction}

Fungal infection of the nose and paranasal sinuses is an atypical condition that is becoming more common. From the point of view of risk and predisposing factors, the most common are: chronic bacterial sinusitis, HIV infection, allergic rhinosinusitis, long-term corticosteroid and / or antibiotic treatment, poorly controlled diabetes, immunocompromised patients. The diagnosis is based on computed tomography of the paranasal sinuses, along with the histological appearance of a collection of fungal hyphae. Treatment is based on a database of clinical admissions, trials and the patient's condition.

\section{Clinical case}

We present a case of a 44-year-old man who was admitted to the ENT Clinic at University Hospital Kaspela with complains of difficult nasal breathing, mainly expressed on the left side, facial pain and recurrent sinusitis. The patient did not report that suffers any chronic disease and denied having allergies. He was treated conservatively with nasal spray and antibiotics without any significant effect. CT scan shows hypertrophy of the mucosa nasal mucosa, involving mainly the left side of the nose and paranasal sinuses. The sphenoid sinus presented as a single cavity covered by a highly hypertrophied mucosa with increased density up to $77 \mathrm{HE}$ (Fig1). The described changes are characteristic of a chronic recurrent process. In this case, the treat- ment we decided to perform was functional endoscopic sinus surgery, the anterior group of sinuses were gradually opened.With transethmoidal approach the sphenoid sinus was presented and the natural opening was enlarged. Fungal ball was removed, the sinus was flushed and aspirated. A nasal splint was placed, which remained for 14 days, the nasal pack applied for 24 hours. The patient did not have any significant complaints in the postoperative period.

\section{Discussion}

Fungal rhinosinusitis can be classified in the following way:

(1) Invasive fungal sinusitis - presence of fungal hyphae in the mucosa, submucosa, bone or blood vessels of the paranasal sinuses. This type of fungal rhinosinusitis is more common in immunocompromised patients.

(2) Acute invasive fungal sinusitis.

(3) Chronic invasive fungal sinusitis.

(4) Chronic granulomatous invasive fungal sinusitis.

(5) Non-invasive fungal sinusitis - more common immunocompetent patients.

(6) Allergic fungal sinusitis.

(7) Fungal ball (fungal mycetoma)

A properly taken biopsy and silver impregnation of swabs and cultures is the key to make a difference between the mycetoma variations. 
Mycetoma is an accumulation of a dense conglomerate of fungal hyphae in the sinus cavity without the presence of allergic mucin. It is the most common form of fungal rhinosinusitis. In most cases, the maxillary sinus is affected, with the sphenoid sinus being second. Most of the patients with this condition are immunocompetent, in addition it is more common in females than in males. In most cases, it is caused by Aspergillus fumigatus. Bipolaris, Curvularia lunata, Alternaria, Pseudallescheria boydii, Rhizopus can also be found.

The etiopathogenesis of sinus mycetoma can be iatrogenic, for example in dental procedures. Aerogenic - inhalation of spores over a long period of time. Another mechanism - impaired mucociliary clearance.

Clinical presentation - usually manifested by the following: facial pain, and frequent nasal secretion. Nasal obstruction is another characteristic symptom. Hyposmia and cacosmia, headache, visual disturbances - for example diplopia (in sphenoidal mycetoma), it is often associated with invasive forms with a bone defect, which portends a poor prognosis for vision. An asymptomatic course of the disease should not be ruled out. The size and pneumatization of the sphenoid sinus vary and many important structures lie in close proximity (cavernous sinus, pituitary gland,internal carotid artery, cranial nerves II, III, IV, V1, V2, and VI). Due to these structural features, sphenoid sinusitis can cause serious complications, including headaches and visual disturbances.

\section{Diagnosis}

The diagnosis is based on computed tomography of the paranasal sinuses, along with the histological appearance of a collection of fungal hyphae. In many cases, the fungal elements in the mycetoma are not viable and cultural examinations of surgical material are negative. In $70 \%$ of mycetomas, the diagnosis is histological. Soft tissue tumors should be considered in the differential diagnosis. CT data for sinus mycetoma. X-ray data for sinus shadowing with / without associated calcifications. Mucopurulent materials with the appearance of cheese. Matte, dense conglomerate of fungal ball separated from but adjacent to the sinus respiratory mucosa. Chronic inflammatory process of the mucosa adjacent to fungal substances. No histological evidence of fungal invasion of the mucosa, associated blood vessels or underlying bone, microscopically visualized by Gamori methanamine silver swab. Hypodensity at $\mathrm{T} 1$ and lack of signal at T2 (due to the presence of calcium, air and iron-magnetic elements). In both series, T1 and $\mathrm{T} 2$, peripheral amplification is observed.

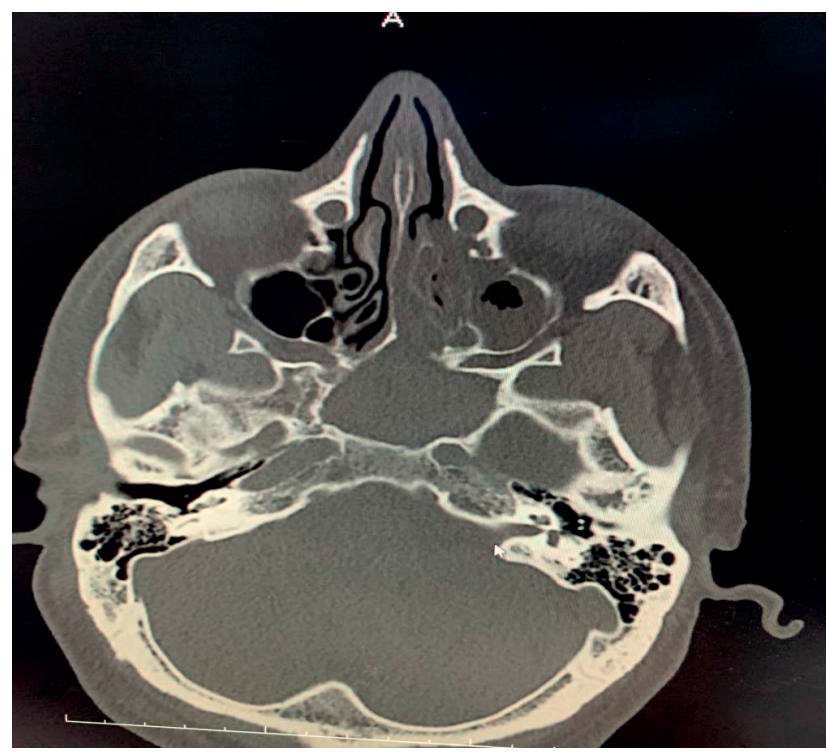

Figure 1. The sphenoid sinus presented as a single cavity covered by a highly hypertrophied mucosa with increased density up to $77 \mathrm{HE}$

\section{Treatment}

The most important part of the treatment is to remove the fungal ball and restore the normal aeration of the sinus/es. Extensive sphenoidotomy during the sphenoetmoidal recess followed by extensive irrigation and mucosal biopsy (Fig. 2) and (Fig. 3). There are no clear indications for antifungal drug therapy in people with normal immunity. Amphotericin B followed by voriconazole. Corticosteroids, such as dexamethasone, are also used. Recurrent cases are rare.

\section{In conclusion}

Fungal infection should be considered in the differential diagnosis in patients with chronic sinusitis who do not respond to antibiotic treatment, as well as in those with unilateral sinus involvement. 


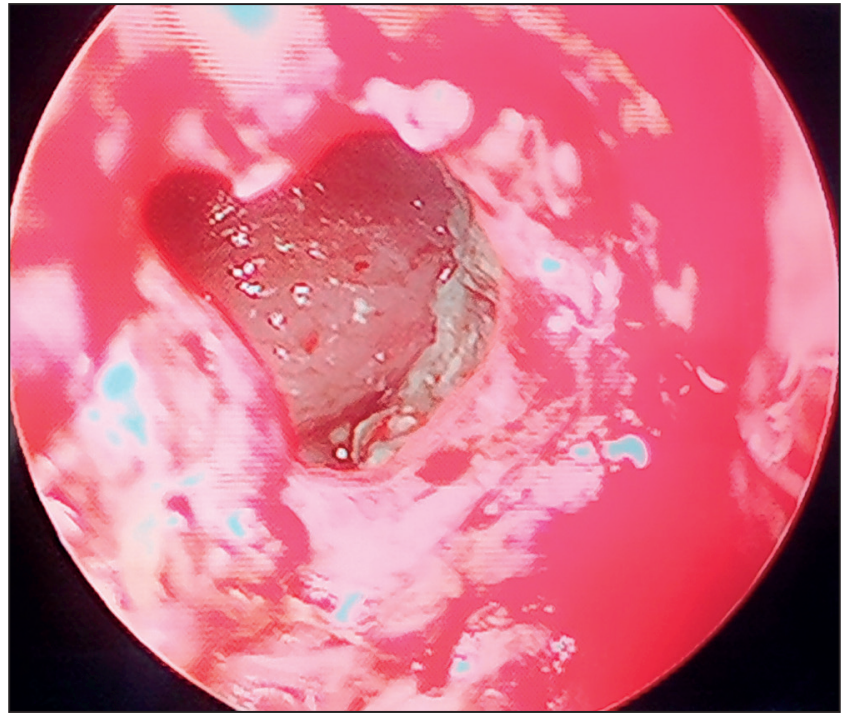

Figure 2. Extensive sphenoidotomy during the sphenoetmoidal recess.

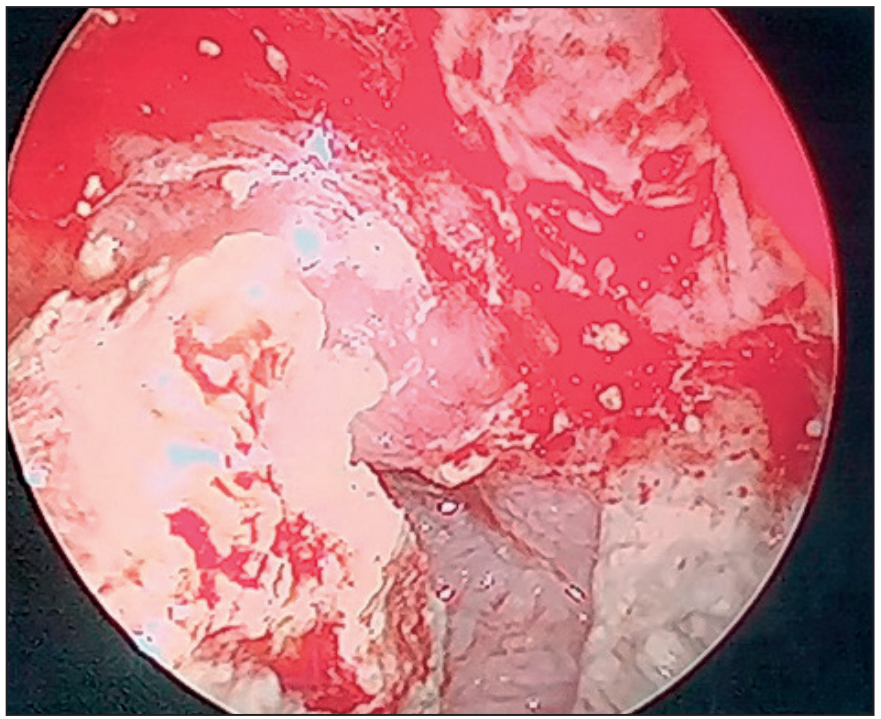

Figure 3. Extensive irrigation and mucosal biopsy

\section{References:}

1. Stammberger M, Jakes R, Beaufort F, Austria G. Aspergillosis of the Paranasal Sinuses, X-ray Diagnosis, Histopathology and Clinical Aspects. Ann Otol Rhinol Laryngol. 1984;93:251-256.

2. Hartwick RW, Batsakis JG. Pathology consultation. Sinus Aspergillosis and allergic fungal sinusitis. Ann Otol Rhinol Laryngol. 1991;100:427430.

3. Bradwein M. Histopathology of sinonasal fungal disease. Otolaryngol Clin North Am. 1993;26:949-981.

4. Stevens M. Primary fungal infections of the paranasal sinuses. Am J Otolaryngol. 1981;2:348-357.

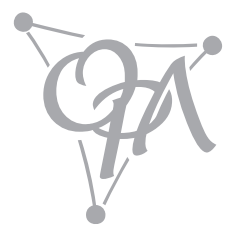

